\title{
Fallbericht zu einer seltenen Berufskrankheit: Eine zu Lebzeiten nicht anerkannte Talkose
}

\author{
Case Report of a Rare Occupational Disease: A During Life Non-Recognised \\ Occupational Disease - Talcosis
}

Autoren

Institute
V. Neumann ${ }^{1 *}$, F. Schulz ${ }^{2 *}$, A. Theile ${ }^{3}$, S. Löseke ${ }^{1}$, K. Püschel ${ }^{2}$, A. Tannapfel ${ }^{1,3}$

Deutsches Mesotheliomregister am Institut für Pathologie der Ruhr-Universität Bochum am Berufsgenossenschaftlichen Universitätsklinikum Bergmannsheil (Direktorin: Professor Dr. med. Andrea Tannapfel)

2 Institut für Rechtsmedizin, Universitätsklinikum Hamburg-Eppendorf, Hamburg (Direktor: Professor Dr. med. Klaus Püschel)

3 Institut für Pathologie der Ruhr-Universität Bochum am Berufsgenossenschaftlichen Universitätsklinikum Bergmannsheil.

(Direktorin: Professor Dr. med. Andrea Tannapfel) eingereicht 5.2.2011

akzeptiert nach Revision 18.2.2011

\section{Bibliografie}

DOI http://dx.doi.org/ $10.1055 / \mathrm{s}-0030-1256286$

Online-Publikation: 16. 3. 2011

Pneumologie 2011; 65:

471-476 @ Georg Thieme

Verlag KG Stuttgart · New York ISSN 0934-8387

\section{Korrespondenzadresse \\ Diplom-Biologe \\ Volker Neumann}

Deutsches Mesotheliomregister am Institut für Pathologie Bürkle-de-la-Camp-Platz 1 44789 Bochum

Volker.Neumann@ruhr-unibochum.de

\section{Zusammenfassung \\ $\nabla$}

Talk ist ein hydratisiertes Magnesiumsilikat, das in der chemischen, kosmetischen, Leder-, Papierund Bauindustrie Verwendung findet. Eine interstitielle Lungenerkrankung - Talkose -, die auf eine reine Talkumexposition zurückgeht, ist eine seltene Pneumokoniose. Häufiger finden sich Lungenerkrankungen durch die intravenöse Gabe von Talk infolge eines Drogenmissbrauchs. Auch kann Talkum mit Asbest oder Quarz verunreinigt sein und es treten Asbest- oder quarzbedingte interstitielle Lungenerkrankungen auf. Wir stellen hiermit einen Fall über einen Arbeiter vor, der bei der Reifenherstellung beruflich gegenüber Talkum exponiert war. Zu Lebzeiten war eine Berufskrankheit nicht anerkannt. Bei dem Verstorbenen wurde erst auf dem Weg zur Einäscherung durch den Rechtsmediziner (in amtsärztlicher Funktion) im Rahmen der zweiten Leichenschau der Verdacht auf eine Berufskrankheit geäußert und eine Obduktion veranlasst. Im Rahmen der Obduktion konnte eine fortgeschrittene Lungenfibrose mit teils wabenartigem Lungenumbau nachgewiesen werden. Es fanden sich massenhaft eingelagerte kristalline, unter polarisiertem Licht stark doppelbrechende Partikel. Licht- und elektronenmikroskopisch fanden sich keine Hinweise auf eine erhöhte pulmonale Asbestbelastung oder eine Silikose. Die Analysen konnten Fasern und Partikel mit der Zusammensetzung von Magnesium und Silizium nachweisen und diese eindeutig als Talkum klassifizieren. Basierend auf den pathologisch-anatomischen Untersuchungen, den Ergebnissen der mineralogischen Untersuchungen, der nachgewiesenen beruflichen Talkumexposition und dem Ausschluss anderer Ursachen für die Induktion der Lungenfibrose konnte die Diagnose einer durch Talkum bedingten interstitiellen Lungenerkrankung gestellt werden. Dieser Fall zeigt deutlich die Relevanz von pathologisch-anatomischen Untersu-

\section{Abstract \\ $\nabla$}

Talc is a hydrated magnesium silicate used in the chemical, ceramic, cosmetic, leather, paper and building industries. Interstitial lung disease - talcosis - due to exclusive talc inhalation is a rare form of pneumoconiosis. More often, pulmonary disease due to talc is encountered after intravenous administration of talc during drug abuse. Talc can contain asbestos or quartz particles which induce asbestosis or silicosis. Here we present a case report about a worker who was exposed to talcum during his work in tyre manufacturing. During his lifetime an occupational disease was not recognised. The deceased had been forwarded to cremation; the legally prescribed second inspection of the corpse induced the suspicion of an occupational disease and an autopsy was ordered. The autopsy revealed a lung fibrosis with honeycomb lung alterations and under polarised light a massive burden with birefringed crystalline particles could be visualised. Light and electron microscopic lung dust analyses could exclude an elevated asbestos lung burden. The element analysis of foreign body material in lung tissue confirmed its chemical composition of magnesium and silicon which was consistent with talc. Based on the pathological and mineralogical findings, the confirmed occupational exposure towards talc and, due to the exclusion of other possible causes (asbestos, quartz), the diagnose of a talc-induced interstitial lung fibrosis talcosis - was established. This case emphasises the importance of pathological-anatomic examinations in combination with lung dust analysis to reveal occupational exposure as a cause of an interstitial lung disease.

\footnotetext{
* Herr Dr. Schulz und Herr Neumann haben in den gleichen Maßen zu dem Manuskript beigetragen.
} 
chungen und der Lungenstaubanalytik, um eine berufliche Exposition als Ursache für die Entwicklung einer interstitiellen Lungenerkrankung nachzuweisen.

\section{Einleitung}

Aus mineralogischer Sicht handelt es sich bei Talk (Speckstein) um ein „weiches“, natürlich vorkommendes Mineral (Magnesiumsilikat mit einer idealen chemischen Zusammensetzung von: $63 \% \mathrm{SiO}_{2}, 31,7 \% \mathrm{MgO}$ und 4,8\% $\mathrm{H}_{2} \mathrm{O}$ ), das in Form von Plättchen oder auch in Faserform auftritt [1,2].

Da Talk, Quarz und Asbest in den gleichen geologischen Schichten vorkommen, können je nach Abbaugebiet Verunreinigungen von Talk mit Asbestfasern oder Quarz vorliegen. Die Feinstruktur von Talk wird in der Regel als plättchenförmig beschrieben, es kommen aber auch hohe Gehalte an Talkfasern (1 Million Talkumfasern pro mg) vor [3].

Talkum stellt aufgrund seines breiten Anwendungsspektrums ein industriell häufig verwendetes Mineral dar. In über 500 verschiedenen Produkten wird Talk in der Papierindustrie, bei der Seifenherstellung, in der Leder- und Gummiindustrie (Füllstoff, Trennmittel), in der Reifenherstellung, in Metallgießereien, in der chemischen Industrie (Farbträger), im kosmetischen Bereich (Puder) und im medizinischen Bereich (Handschuhe) verwendet $[1,4]$.

Die Erstbeschreibung einer Talkum-assoziierten Pneumokoniose erfolgte bereits im Jahr 1896 [5]. Da nach einer Exposition gegenüber quarz- und asbestfreiem Talkum wiederholt Lungenfibrosen mit massiven Einlagerungen von Talkumpartikeln nachweisbar waren, wird davon ausgegangen, dass auch reines Talkum eine fortschreitende Lungenfibrosierung unterhalten kann [1].

Radiologisch findet sich eine diffuse Fibrose der Lungen mit Trübung der Mittel- und Unterfelder. Pathologisch-anatomisch lassen sich Granulombildungen, mehrkernige Riesenzellen und diffuse Lungenfibrosen mit Ausbildung einer Wabenlunge beobachten [1]. Histologisch sind interstitielle Ablagerungen mit plättchenförmigen, stark doppelbrechenden Partikeln in den Fibrosierungszonen charakteristisch.

Von den Pneumokoniosen, die durch die Inhalation von Talk bedingt sind, müssen pulmonale Erkrankungen durch Talk abgegrenzt werden, die auf eine intravenöse Zufuhr zurückzuführen sind $[4,6-8]$. So wurden wiederholt pulmonale Talk-assoziierte Erkrankungen beschrieben, die bei Patienten mit intravenösem Drogenkonsum auftreten [9-11]. In diesen Fällen wurden talkhaltige Tabletten, die zur oralen Aufnahme vorgesehen waren, intravenös appliziert. Als Folge dieser Talkumaufnahme wurden arterielle Obstruktionen und Granulombildungen nachgewiesen $[6,7]$.

Damit lassen sich 4 unterschiedliche pulmonale Erkrankungen unter Talkumbeteiligung abgrenzen [12]:

1. Talkum-Silikose (Talcosilicosis)

2. Talkum-Asbestose (Talcoasbestosis)

3. Talkose (Talcosis)

4. Talkum-bedingte Lungenerkrankung durch intravenöse Applikation von Talkum

\section{Fallbericht}

$\nabla$

\section{Vorgeschichte}

Der Patient war ab 2001 wiederholt in stationärer Behandlung. Im Dezember 2001 erfolgte eine Bypassoperation. Radiologisch kam eine interstitielle Zeichungsvermehrung in beiden Lungenmittel- und -unterfeldern zur Darstellung.

Im April 2008 wurden im Rahmen einer stationären Behandlung die Diagnosen einer Anämie, einer Belastungsluftnot, einer Refluxösophagitis, einer chronisch atrophischen Gastritis, eines Nicht-ST-Hebungsinfarktes im Rahmen der Anämie bei koronarer Herzkrankheit mit guter linksventrikulärer Funktion und eines intermittierenden Vorhofflimmerns gestellt. Ferner bestand der Verdacht auf eine Lungenfibrose. Es erfolgte die Beendigung der Antikoagulation mit Marcumar wegen gastrointestinaler Blutungen.

Bei weiteren stationären Aufenthalten im Jahre 2008 wurde eine ausgeprägte Fundusgastritis diagnostiziert und Blutkonserven zur Behandlung einer Anämie gegeben. Weiterhin erfolgte eine Kortisonstoßtherapie mit Verbesserung des respiratorischen $\mathrm{Zu}$ stands.

Erstmals im August 2009 wurde ein Pneumologe in die Behandlung miteinbezogen. Dieser stellte die Diagnose einer Lungenfibrose, einer sekundären pulmonalen Hypertonie und einer chronischen respiratorischen Insuffizienz. Die Blutgasanalyse zeigte Werte von $\mathrm{pO}_{2} 57,0 \mathrm{mmHg}, \mathrm{pCO}_{2} 32,7 \mathrm{mmHg}, \mathrm{pH} 7,41$ und $\mathrm{ABE}$ $2,6 \mathrm{mmol} / \mathrm{l}$. In Ruhe zeigte sich eine deutliche Partialinsuffizienz trotz Hyperventilation. Nach 15 Minuten $\mathrm{O}_{2}$-Gabe zeigte sich eine deutliche Verbesserung der Oxygenierung $\left(\mathrm{pO}_{2} 66,9 \mathrm{mmHg}\right.$, $\mathrm{pCO}_{2}$ 27,5 mmHg, pH 7,53 und ABE 2,0 mmol/l). Es wurde eine ambulante Sauerstofftherapie eingeleitet.

Im Januar 2010 erfolgte die nächste Vorstellung beim Pneumologen. Neben der Diagnose einer schweren pulmonalen Hypertonie bei Mehrklappenvitium wurde die Diagnose einer „Talkum-Lunge“ gestellt. Die Blutgasanalyse zeigte Werte von $\mathrm{pO}_{2} 46,2 \mathrm{mmHg}$, $\mathrm{pCO}_{2}$ 33,6 mmHg, pH 7,46 und ABE 0,5 mmol/l. In Ruhe zeigte sich eine schwere Partialinsuffizienz trotz Hyperventilation. Die Sauerstofftherapie wurde weitergeführt.

Im Juli 2010 verstarb der Patient im Alter von 84 Jahren. Auf der Todesbescheinigung war eine respiratorische Insuffizienz bei Lungenfibrose vermerkt. Obwohl im Januar 2010 als Diagnose eine „Talkumlunge“ aufgeführt wurde, erfolgte keine ärztliche Anzeige auf eine Berufskrankheit.

Der Verstorbene wurde für die Einäscherung vorbereitet. Der die amtsärztliche Leichenschau durchführende Rechtsmediziner stoppte die Einäscherung und veranlasste eine Obduktion und ergänzende Untersuchungen zur Aufklärung der Genese der Lungenfibrose. Zu diesem Zeitpunkt waren weder die gestellte Diagnose einer „Talkumlunge“ noch die beruflichen Expositionsverhältnisse bekannt.

\section{Berufsanamnese}

Da die Verdachtsanzeige auf das Vorliegen einer Berufskrankheit erst nach dem Tod des Versicherten an die Berufsgenossenschaft übermittelt wurde, starteten die Erhebungen der Präventionsabteilung erst post mortem. Durch Befragung der Angehörigen konnte schnell eruiert werden, dass der Versicherte bei der Reifenherstellung 1965 bis 1973 gegenüber Talkum exponiert war. Der Stellungnahme der Präventionsabteilung vom Oktober 2010 ist zu entnehmen, dass der Patient vom 4.10.1965 bis 31.1.1973 in der Reifenherstellung tätig war. Der Versicherte arbeitete als Rotationspressenführer und Heizer im Vulkanisierungsbereich. 


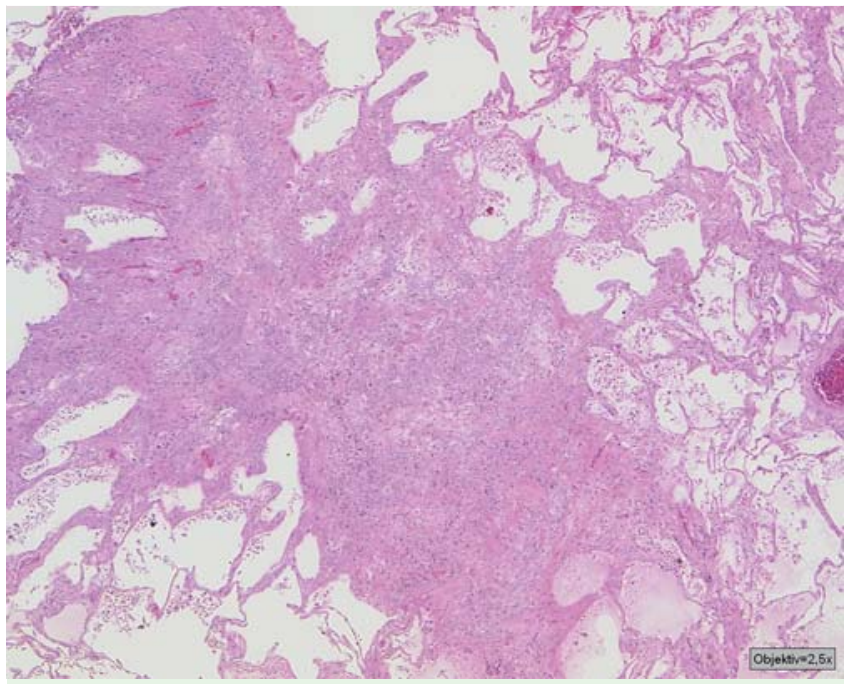

Abb. 1 Histologische Aufnahme des Lungenparenchyms mit ausgeprägtem fibrotischem/granulomatösem Umbau und zahlreich eingelagerten Partikeln (25×, HE-Färbung).

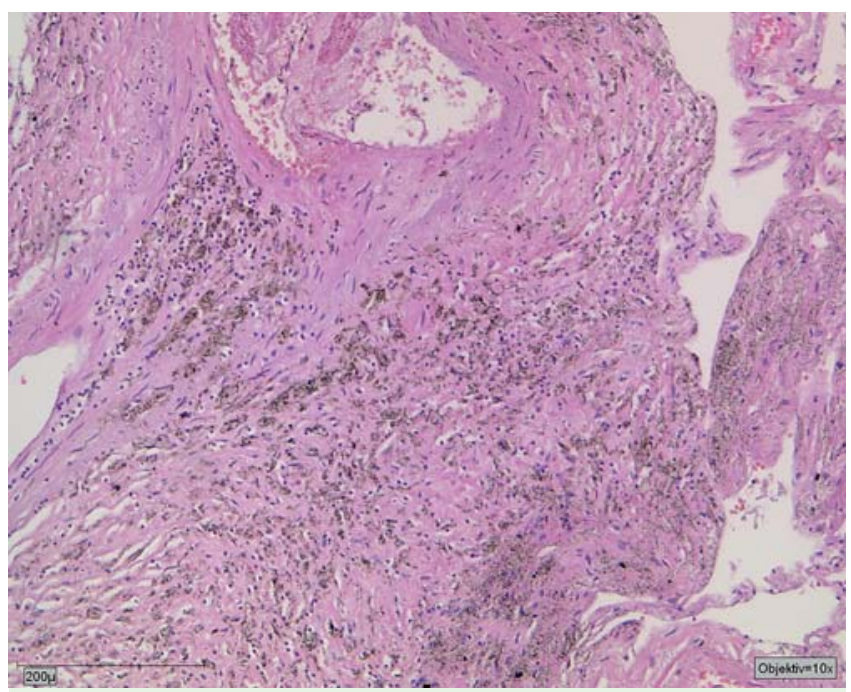

Abb. 2 Lungenparenchym mit ausgeprägtem fibrotischem Umbau und zahlreich eingelagerten Talkumpartikeln (100×, HE-Färbung).

In diesem Arbeitsfeld wurden Gummirohmischungen vorgenommen und im großen Umfang Talkum verwendet. Nach den Ermittlungen der Präventionsabteilung bestanden eine direkte und auch eine indirekte (Bystander) Exposition gegenüber Talkum. Die Staubkonzentration in diesem Arbeitsbereich lag bei bis zu $55 \mathrm{mg} / \mathrm{m}^{3}$. Basierend auf der Annahme, dass das Talkum mit Asbestfasern verunreinigt war, wurde eine kumulative Dosis von 1,5 Asbestfaserjahren (4.10.1965 bis 31.1.1973) veranschlagt.

\section{Sektionsergebnisse}

Im Rahmen der Obduktion in der Rechtsmedizin konnte eine deutliche pulmonal bedingte Kachexie, eine diffuse Fibrose beider Lungen mit teils emphysematösem Lungenumbau, eine nicht ganz frische Thrombose im Hauptstamm der linken Lungenarterie und in der Schlagader des rechten Lungenunterlappens eine chronische Bronchitis, eine Anthrakose des Lungenfells und eine Hypertrophie des rechten Herzen nachgewiesen werden. Hyaline Pleuraplaques lagen nicht vor. Für einen akuten oder alten Herzinfarkt fand sich kein morphologisches Korrelat. Als Todesursa-

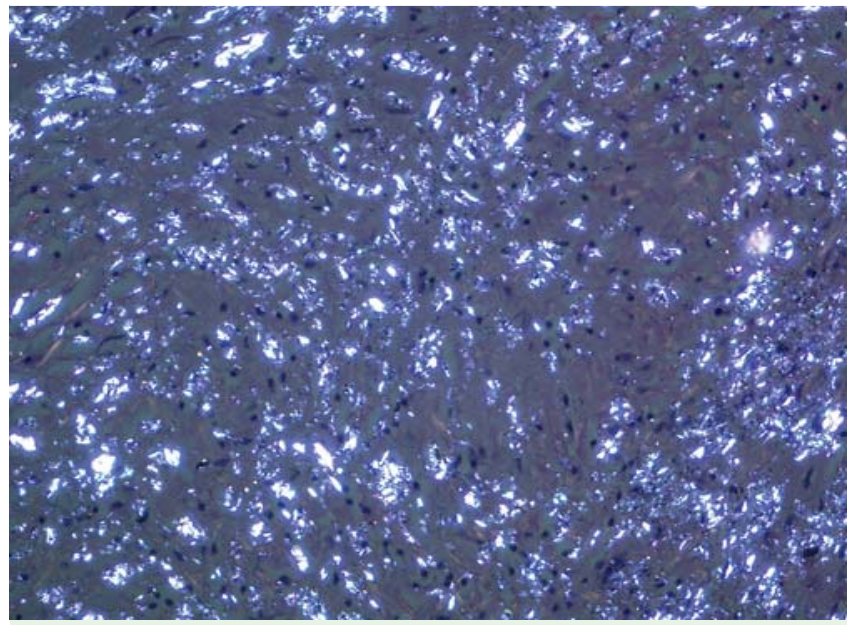

Abb. 3 Lungenparenchym im Polarisationsbild mit zahlreichen eingelagerten stark doppelbrechenden Talkumpartikeln (200×, Pol, HE-Färbung).

che ließ sich ein Rechtsherzversagen bei komplexer Lungenerkrankung (Lungenfibrose und emphysematöser Lungengewebsumbau) bei Lungenarterienthrombose/-embolie mit Verdacht auf Infarktpneumonie bei pulmonaler Kachexie belegen.

Im Anschluss an die Sektion in der Rechtsmedizin folgten ergänzende Untersuchungen in der Pathologie Bochum/Deutsches Mesotheliomregister. Makroskopisch zeigte sich das Bild einer „Destroyed lung" mit einer fortgeschrittenen interstitiellen Lungenfibrose und begleitendem irregulärem teils bullösem Traktionsemphysem, Pleurafibrose, chronischer Bronchitis mit Bronchiektasien, kleinherdiger Bronchopneumonie, Pulmonalsklerose und Ektasie.

\section{Ergebnis der histologischen Untersuchungen}

Hochgradig bindegewebig umgebautes Lungenparenchym mit teils knotigem ( $\boldsymbol{A}$ Abb. 1) und teils wabenartigem Lungenumbau. Es finden sich massive Einlagerungen von graubraunem puderartigem Staub $(\bullet$ Abb. 2).

In Kombination mit den fibrosierenden Lungenveränderungen zeigt sich ein Traktionsemphysem. Polarisationsoptisch finden sich in den Fibrosierungszonen eingelagerte spieß- und plättchenartige, stark doppelbrechende kristalline Fremdpartikel (৫ Abb. 3).

Kein Nachweis von Asbestkörpern. Die stark doppelbrechenden spießförmigen Partikel weisen vorwiegend eine Länge von $>6 \mu \mathrm{m}$ und einen Durchmesser von weniger als $2 \mu \mathrm{m}$ auf und sind damit nach der WHO-Klassifikation (Länge $>5 \mu \mathrm{m}$, Durchmesser $<3 \mu \mathrm{m}$ und Verhältnis Länge zu Durchmesser von $>3: 1$ ) als Talkumfasern einzustufen. Fokal finden sich auch mehrkernige Riesenzellen. Die interstitielle Lungenfibrosierung mit dem Nachweis mikrokristalliner, stark doppelbrechender Fremdpartikel weist das histologische Bild einer Talkum-assoziierten Lungenfibrosierung auf.

\section{Lichtmikroskopische und elektronenmikroskopische Lungenstaubanalytik}

In den vier durchgeführten lichtmikroskopischen Analysen aus dem rechten und linken Lungenober- und -unterlappen konnte keine vermehrte pulmonale Asbestkonzentration nachgewiesen werden. Die ermittelten Konzentrationen waren geringer als 20 Asbestkörper pro Gramm Lungenfeuchtgewebe und lagen somit in dem Bereich von sogenannten Normallungen [13]. 

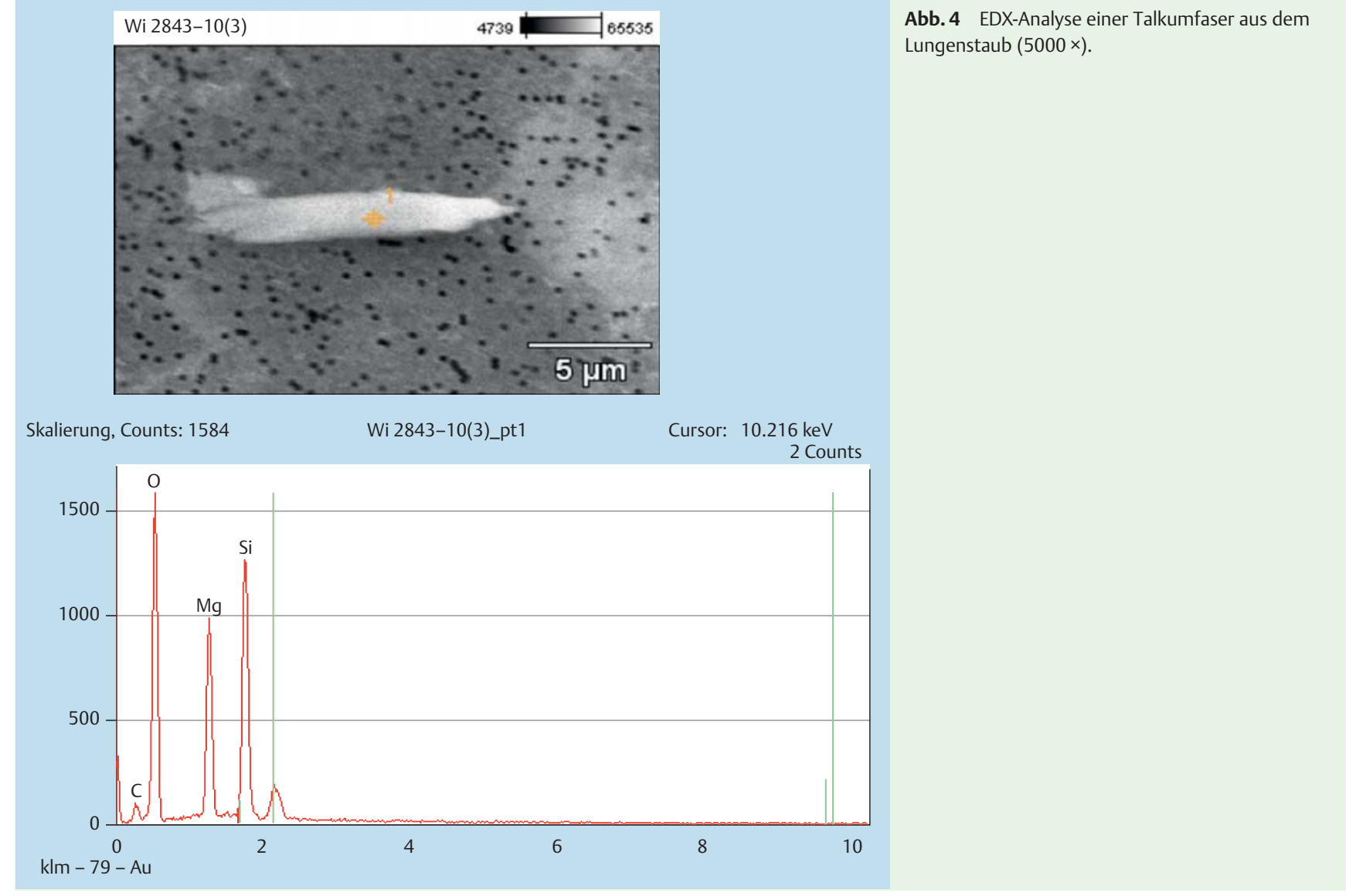

Abb. 4 EDX-Analyse einer Talkumfaser aus dem Lungenstaub $(5000 \times)$.

Weiterhin wurde zusätzlich eine elektronenmikroskopische Lungenstaubanalyse am Feldemissions-Rasterelektronenmikroskop [14] nach Vorschrift der BIA-Arbeitsmappe 26 Lfg. III/0I 7489/ 2001 (Bestimmung von anorganischen Fasern im menschlichen Lungengewebe unter Verwendung eines Feldemissions-Rasterelektronenmikroskops) durchgeführt. Dabei wurden Asbestfasern (Chrysotil oder Amphibol) elektronenmikroskopisch nicht nachgewiesen. Jedoch waren maximal 2560400 faserförmige Talkumfasern (Talkumfasern oder faserförmige Talkumpartikel) pro Gramm Lungenfeuchtgewebe quantifizierbar.

\section{Energiedispersive Röntgenmikroanalyse (EDX)}

In der EDX-Analytik konnte am histologischen Schnittpräparat bei insgesamt 98 Einzelanalysen eine Inkorporation von Talkumpartikeln und Talkumfasern in den Fibrosierungsarealen nachgewiesen werden $(\bullet$ Abb. 4).

Die Talkumpartikel enthalten Magnesium und Silizium als Leitelemente. Der Abgleich mit einer Referenz-Talkumprobe ergibt röntgenmikroanalytisch identische Befunde.

\section{Latenzzeit und Interimszeit}

Aus den vorliegenden Daten zum Expositionszeitraum (19651973) und zum Zeitpunkt der Erstdiagnose der Lungenfibrose (2001) ergeben sich eine Interimszeit von 28 Jahren und eine Latenzzeit von 36 Jahren.

\section{Diskussion $\nabla$}

Das Krankheitsbild einer Talkose gehört zu einer sehr seltenen Variante der Pneumokoniosen. Pneumokoniosen sind dadurch gekennzeichnet, dass sich pathologisch-anatomisch in den Fibrosierungsarealen die dafür verantwortlichen Staubpartikel oder Fasern nachweisen lassen [15].

Bei der Analyse und Untersuchung von Talkum-bedingten Erkrankungen ist insbesondere die Möglichkeit einer Verunreinigung mit Asbestfasern und Quarz mit zu berücksichtigen. Wenn in Talkum Asbestfasern nachgewiesen werden, handelt es sich in erster Linie um Amphibolasbestfasern [16]. Bei der elektronenmikroskopischen Analyse der Zusammensetzung der Staubablagerungen in der Lunge zeigen sich oft Mischstaubeinlagerungen mit Anteilen von Asbest, Glimmer, Talkum, Kaolin und Quarz. Bei derartigen Mischbefunden wurde so z.B. der Asbest- oder der Quarzkomponente die maßgebliche fibrogene Wirkung zuerkannt und die Lungenfibrosen als Mischstaubpneumokoniosen mit Quarz oder Asbest als relevantem fibrogenem Faktor eingestuft $[17,18]$.

Obwohl Talkum nur eine geringgradige fibrogene Potenz zugeschrieben wurde, weisen sowohl Untersuchungen mit asbestverunreinigtem als auch mit asbestfreiem Talkum auf die Entwicklung von fibrosierenden Talkum bedingten Lungenerkrankungen hin $[1,6]$. Während in einer Untersuchung nach einer Exposition gegenüber reinem Talkum mittels der bildgebenden Diagnostik die Ausbildung von Pneumokoniosen nicht beobachtet werden konnte [19], wurden in weiteren Studien mit Kollektiven von Arbeitern in Talkminen (asbesthaltiges Talkum Tremolit, Anthophyllit) und der Talk-verarbeitenden Industrie [20 - 22] Pneumokoniosen und auch pleurale Verdickungen dokumentiert [21]. 
Auch bei Untersuchungen mit Kollektiven, die gegenüber asbestund quarzfreiem Talkum exponiert gewesen waren, konnten radiologisch Pneumokoniosen nachgewiesen werden [22].

Nach den vorliegenden Studienergebnissen werden Talkosen bereits nach kurzen Latenzzeiten bei massiver Talkumexposition beobachtet $[6,23,24]$. Mit Verringerung der Expositionshöhe werden verlängerte Latenzzeiten registriert [6,23,24].

Im vorliegenden Casus finden sich keine Hinweise auf eine andere Ursache als Talkum für die vorliegende Lungenfibrosierung. Weder konnte eine erhöhte Asbest- oder Quarzkonzentration der Lungen nachgewiesen werden noch zeigten sich histologische Veränderungen, die auf eine andere Ursache wie z.B. das Vorliegen einer Sarkoidose $[18,25]$ hingedeutet hätten. Auch Pleuraplaques als Hinweis auf eine asbestbedingte Genese der Erkrankung konnten nicht nachgewiesen werden. Mittels der histologischen und elektronenmikroskopischen Untersuchungen konnte aufgezeigt werden, dass die Talkumfasern die Lungenfibrosierung induziert und unterhalten haben. Durch die Überlastung der Reinigungsmechanismen der Lungen (Overload) mit dem Talkum, welches per se nur eine geringe fibrogene Potenz aufweist [1], wurden im vorliegenden Fall eine persistierende Entzündungsreaktion und eine fortschreitende Lungenfibrosierung induziert.

In Übereinstimmung mit anderen Untersuchungen zeigten sich in diesem Fall auch granulomatöse Veränderungen $[1,15]$. Die hochgradigen Lungenveränderungen mit wabenartigem Lungenumbau zeigen, dass nach Einwirkung von Talkum auch schwergradige Lungenfibrosen auftreten können. Für die Genese einer Talkum-assoziierten Lungenfibrosierung ist die hohe Biobeständigkeit sicherlich von entscheidender Bedeutung.

In Konkordanz mit weiteren Untersuchungen [3] konnte eine hohe Biopersistenz der Talkfasern von mehreren Jahrzehnten belegt werden. Die Talkumfasern konnten in hoher Zahl (über 2,5 Millionen Talkumfasern pro Gramm Lungenfeuchtgewebe) noch 28 Jahre nach Beendigung der Exposition in den Lungen nachgewiesen werden. Talkum weist damit eine hohe Biobeständigkeit auf. Diese liegt in einem Bereich, den auch Amphibolasbestfasern aufweisen. Die Biobeständigkeit ist deutlich höher als bei den nur gering biobeständigen Chrysotilfasern, bei denen Halbwertszeiten von weniger als einem Monat beschrieben wurden [3,26,27]. Leider wurde die ärztliche Anzeige auf eine Berufskrankheit zu Lebzeiten nicht gestellt, obwohl zwei Monate vor dem Tod des Patienten eine „Talkumlunge“ diagnostiziert wurde. Die Ermittlungen der Präventionsabteilung hätten so schon zu Lebzeiten des Patienten aufgenommen werden können.

Erfreulicherweise konnten nach dem Tode des Patienten begonnene Ermittlungen noch relevante Ergebnisse über die stattgehabten Schadstoffbelastungen erbringen. Es trat somit nicht der Fall ein, dass wegen fehlender arbeitstechnischer Ermittlungsergebnisse der vollbeweisliche Nachweis der beruflichen und versicherten Exposition und somit die Anerkennung einer Berufskrankheit erschwert oder unmöglich gemacht wurde. Die reine Möglichkeit einer beruflichen Exposition hätte für die Anerkennung einer Berufskrankheit nicht ausgereicht.

In Deutschland wurde bereits 1952 die Talkose unter die BK Nr. 4101 subsumiert. In der amtlichen Begründung zur 5. Verordnung vom 26.07.1952 wird ausgeführt, „dass in der erläuternd beigefügten Bezeichnung „Silikose“ alle Veränderungen und $\mathrm{Zu}$ stände des Lungengewebes einzuschließen sind, „die unter dem Einfluss der fibroplastischen Wirkung gelöster Kieselsäure entstehen, einerlei ob diese ursprünglich aus freier $\mathrm{SiO}_{2}$ oder gebundener Kieselsäure (Silikaten) stammte, also außer der Silikose im engeren kli- nisch-anatomischen Sinne (Quarzlunge) auch die gemein als „Silikatose" bezeichneten, Knötchen und Schwielen bildenden Staublungenerkrankungen, wie die der Silikose im engeren Sinne entsprechenden knotigen (nodösen) Staublungenerkrankungen durch Talkum, Glimmer, Kieselgur“ [15].

Für den Umgang mit Talkum wurden Grenzwerte für die maximale Belastung am Arbeitsplatz geschaffen. Für Talkum gilt ein MAK-Wert (Maximale Arbeitsplatz-Konzentration) von $2 \mathrm{mg} / \mathrm{m}^{3}$ Feinstaub.

Zwischenzeitlich wurden eine Berufskrankheit und Hinterbliebenenleistungen seitens der Unfallversicherung anerkannt.

Aus diesem Beispiel dieser zu Lebzeiten nicht angezeigten Berufskrankheit lässt sich folgern:

1. Es gibt eine Dunkelziffer im Bereich der Berufskrankheiten. Sowohl internationale Studien als auch die Erfahrungen im Deutschen Mesotheliomregister lassen diesen Schluss zu $[13,14]$.

2. Im vorliegenden Casus kam dem in amtsärztlicher Funktion tätigen Rechtsmediziner - im Rahmen der zweiten Leichenschau - vor der Feuerbestattung die Rolle des letzten Zeugen für den Patienten zu. Wenn auf einem Totenschein eine nicht näher spezifizierte „Lungenfibrose“ aufgeführt wird, ist zu empfehlen, dass weitere Nachforschungen veranlasst werden. Ggf. sollte eine ergänzende Untersuchung im Rahmen einer Obduktion eingeleitet werden.

\section{Interessenkonflikt}

$\nabla$

Die Autoren geben an, dass kein Interessenkonflikt im Sinne der Richtlinien des International Committee of Medical Journal Editors besteht.

\section{Literatur}

1 Gibbs AE, Pooley F, Griffiths D et al. Talc pneumoconiosis. A pathological and mineralogic study. Hum Pathol 1992; 23: 1344-1354

2 Vallyathan N, Craighead J. Pulmonary pathology in workers exposed to nonasbestiform talc. Hum Pathol 1981; 12: 28-35

3 Rödelsperger K, Brückel B, Arlhelger R et al. Beständigkeit langer Asbestund Talkumfasern in der menschlichen Lunge. Zbl Arbeitsmed 1995; 45: $410-421$

4 van Huisstede A, Hegt V, Otte-Holler I et al. Talcosis due to abundant use of cosmetic talcum powder. Europ Respir Rev 2010; 116: 165-168

5 Thorel $C$. Talc lung. A contribution to the pathological anatomy of pneumoconiosis. Beitr Pathol Anat Allgem Pathol 1896; 20: 85

6 Roberts WC. Pulmonary talc granulomas, pulmonary fibrosis and pulmonary hypertension resulting from intravenous injection of talc containing drugs intended for oral use. Proc 2002; 15: 260-261

7 Püschel $K$, Schoof $W$. Zur Morphogenese hämatogen entstandener Fremdkörpergranulome im Lungenparenchym. Beitr Gericht Med 1987; 45: $121-128$

8 Püschel K, Schmoldt A. Drogennot- und -Todesfälle. In: Madea D, Brinkmann D Hrsg. Habbuch Gerichtliche Medizin 2. Berlin Heidelberg: Springer; 2004

9 Hopkins G, Taylor D. Pulmonary talc granulomatosis. Ann Rev Repir Dis 1970; 101: $101-108$

10 Arnett E, Battle W, Russe J. Intravenous infection of talc containing drugs intended for oral use: A cause of pulmonary granulomatosis and pulmonary hypertension. Am J Ind 1976; 60: 711 - 717

11 Robertson C, Reynolds J, Wilson J. Pulmonary hypertension and foreign body granulomatosis, in intravenous drug abusers: Documentation by cardiac catheterization and lung biopsy. Ann J Med 1976; 61: 657-663

12 Feigin $D$. Talc: Understanding its manifestation in the chest. Am Respir 1985; 146: 295-301

13 Neumann $V$, Kraus T, Fischer $M$ et al. Wertigkeit von pathologisch anatomischen und staubanalytischen Untersuchungen bei asbestassoziierten Berufskrankheiten am Beispiel der BK 4104. Pneumologie 2009; 63: $588-593$ 
14 Neumann V, Löseke S, Tannapfel A. Asbestassoziierte Erkrankungen: Was leistet die Lungenstaubanalytik bei der Bewertung einer Berufskrankheit nach Nr. 4103 und 4104 der BKV? ASU 2010; 45: 104-115

15 Worth G. Die Pneumokoniosen: Die Talkumlunge. In: Worth G, Schiller E Hrsg. Die Pneumokoniosen. Geschichte, Pathogenese, Morphologie, Klinik und Röntgenologie. Köln: Staufen; 1954

16 Mattenklott M. Asbest in Talkumpudern und Speckstein - heutige Situation. Gefahrstoff Reinh Luft 2007; 7/8: 287-292

17 Lockey J. Nonasbestos fibrous minerals. Clin Chest Med 1981; 2: 203 218

18 Tukianinen P, Nickels J, Taskinen E et al. Pulmonary granulomatous reaction: talc pneumoconiosis of chronic sarcoidosis? Brit J Ind Med 1984; 41: 84-87

19 Hildick-Smith G. Talc recent epidemiology studies. In: Walton W, McGovern E Hrsg. Inhaled particles; 4 ed. Oxford: Pergamon Press; 1977

20 Kleinfeld M, Messite J, Kooyman $O$ et al. Mortality among talc miners and millers in New York State. Arch Environ Health 1967; 14: 663-667
21 Gamble J, Fellner W, Dimeo M. An epidemiology study of a group of talc workers. Am Rev Respir Dis 1979; 119: 741 - 753

22 Wegmann $D$, Peters J, Boundy $M$ et al. Evaluation of respiratory effects in miners and millers exposed to talc free asbestos and silica. Brit J Ind Med 1982; 39: 233 - 238

23 Alivisatos G, Pontikakis A, Terzis B. Talcosis of unusually rapid development. Brit J Ind Med 1955; 12: 43-49

24 Gamble J, Greife A, Hamcock J. An epidemiological-industrial hygiene study of talc workers. Ann Occup Hyg 1982; 26: 841 - 859

25 Gysbrechts C, Michiels E, Verbeken E et al. Interstitial lung disease more than 40 years after a 5 year occupational exposure to talc. Eur Respir J 1998; 11: $1412-1415$

26 Case B. Biological indicators of chrysotile exposure. Ann Occup Hyg 1994; 38: 503-518

27 Churg A. Deposition and clearance of chrysotile asbestos. Ann Occup Hyg 1994; 38: 625-633 